\title{
CUATRO NUEVAS ESPECIES CHILENAS DEL GENERO TRUPANEA SHRANK (DIPTERA, TEPHRITIDAE)
}

DANIEL FRIAS L.

\section{ABSTRACT}

Four new species of Trupanea from Chile are described in this paper: Trupanea foliosi, Trupanea chrysanthemifolii, Trupanea footei and Trupanea thuriferae. Morphological and chromosomal characters of adults and immature stages are used in the descriptions and native host plants of the species are also reported.

\section{INTRODUCCION}

El género Trupanea Shrank, en la zona Neotropical, está constituído por 58 especies de las cuales 14 se distribuyen en Chile (Foote, 1967). Las principales contribuciones acerca de la taxonomía de las especies de Trupanea descritas para Chile corresponden a las de Macquart, 1843; Schiner, 1868; Brèthes, 1908; Hendel, 1914; Malloch, 1933; Hering, 1936, 1941, 1942; Aczél, 1949, 1953; Stuardo, 1946. Los límites genéricos de Trupanea han sido discutidos por Munro, 1964, y el catálogo más reciente corresponde al realizado por Foote, 1967.

Como consecuencia de colectas periódicas de dípteros Tephritidae en el Sur, Zona Central y Norte de Chile, se logró identificar varias especies pertenecientes a diferentes géneros de distribución Chilena entre las cuales se incluyen 4 nuevas especies de Trupanea cuya descripción se realiza en el presente trabajo. Además de la descripción morfológica de los adultos y estados inmaduros, se describen las plantas huéspedes y cariotipos de cada especie.

\section{MATERIAL Y METODOS}

Los ejemplares que se utilizaron en este trabajo estan depositados en la colección del Departamento de Biología Celular y Genética (DBC) de la Universidad de Chile.

Las mediciones corporales, los esquemas de adultos y estados inmaduros se realizaron con cámara clara bajo una lupa estereoscópica Wild M 5. La terminología usada se basa en la descrita por Bush, 1966 y Foote, 1980. Las descripciones se realizaron según el Código Internacional de Nomenclatura Zoológica, 1964. Tanto en machos como en hembras de cada espécie se realizaron las siguientes medidas corporales:

L.C.: Longitud de la cabeza: medida de perfil desde la base de la antena hasta el vértice de la frente.

A.C.: Amplitud de la cabeza: máxima amplitud medida en vista frontal.

A.L.C.: Altura de la cabeza: medida de perfil desde el margen inferior de la gena hasta el vértice de la frente.

A.O.: Amplitud del ojo: máxima amplitud medida en vista fronto lateral de modo que ambos márgenes laterales del ojo aparezcan en el foco.

A.O.L.: Altura del ojo: máxima altura medida en vista fronto lateral.

A.F.: Amplitud de la frente: corresponde a la distancia máxima entre el márgen de cada ojo medida en vista dorsal. 
L.A.: Longitud de la antena: medida en vista frontal desde el márgen inferior del primer artejo al fin del 3er antenito.

L.C.: Longitud de la cara: medida frontalmente.

L.T.: Longitud del tórax: máxima longitud medida en vista dorsal desde la región más anterior del pronoto hasta el ápice del escutelo.

L.A.L.: Longitud del ala: medida desde la base del sector radial hasta el final de la vena $r 4+5$.

A.A.: Amplitud del ala: máxima amplitud medida a la altura de la vena subcostal.

L.A.B.: Longitud del abdómen: en los machos desde el ler tergito abdominal al protandrio. En las hembras desde el 1er tergito abdominal hasta el final del VI tergito abdominal.

L.F.O.: Longitud de la funda del ovipositor.

L.O.: Longitud ovipositor en las hembras.

Los cromosomas se obtuvieron por el método clásico de aplastamiento de células del ganglio nervioso en larvas y gónadas en los adultos. La tinción se realizó con orceína acética. Los cromosomas se midieron en un microscopio Leitz con ocular graduado, confeccionandose los idiogramas según lo propuesto por Boyes et al., 1971. La morfología cromosómica se determinó según la nomenclatura propuesta por Levan et al., 1964.

Trupanea foliosi, sp. $\mathrm{n}$.

(Figs. 1, 5, 9, 13, 14, 21, 25, 29, 33, 37, 41, 43, 46, 49, 52, 55, 59, 60, 64)

Tipos: holotipo hembra, Valparaíso (Mirasol) 3 Febrero de 1981. Paratipos 5 hembras, 6 machos, Valparaíso (Mirasol) 3 Febrero 1981.

Descripción

Las mediciones corporales de los adultos se indican en las Tablas 1 y 2 . Los datos cromosómicos se muestran en la Tabla 5.

Cabeza

Cara amarilla; frente café; triángulo ocelar gris. Cuatro pares de cerdas fronto-orbitales inferiores. Dos pares de cerdas fronto-orbitales superiores siendo el par posterior blanco y más corto que el par anterior. Cerdas verticales internas de color negro. Cerdas verticales externas, post-ocelares, post-verticales y post-oculares de color blanco. Márgenes de la región postocular amarillos con la región central negra (Fig. 1). Antenas como en la Fig. 5 .

Tórax

Gris, cubierto de pequeñas cerdas blancas, cerdas escapulares y propleurales blancas. Un par de cerdas acrosticales. Un par de cerdas dorsocentrales. Escutelo con dos cerdas escutelares. Dos cerdas notopleurales siendo la posterior blanquecina y más corta que la anterior. Mesopleuron cubierto de cerdas pequeñas blancas, una cerda mesopleural negra. Cerdas pteropleurales blancas. Esternopleuron cubierto de pequeñas cerdas blancas; una cerda esternopleural (Fig. 9).

\section{Abdómen}

Gris, cubierto de pequeñas cerdas blancas. Hembras: en el último tergito abdominal con tres pares de cerdas negras. Machos: en el último tergito abdominal con 3 a 4 pares de cerdas negras. En las hembras funda del ovipositor negro cubierto de cerdas pequeñas blancas, excepto en la parte apical donde está cubierto de pequeñas cerdas negras (Figs. 13 y 14).

Patas

Tanto en machos como en hembras totalmente amarillas. Coxas cubiertas de pequeñas cerdas blancas, fémur, tibias y tarsos provistos de cerdas negras. 


\begin{tabular}{|c|c|c|c|}
\hline N & 10 & & 10 \\
\hline L.C. & $\begin{array}{c}0.75+0.12 \\
(0.63 \div 0.85)\end{array}$ & $\begin{array}{r}0.63 \\
(0.59\end{array}$ & $\begin{array}{l}+0.04 \\
-0.67)\end{array}$ \\
\hline A.C. & $\begin{array}{c}0.95 \pm 0.40 \\
(1.00 \div 1.37)\end{array}$ & $\begin{array}{r}1.17 \\
(1.07\end{array}$ & $\begin{array}{l} \pm 0.06 \\
-1.22)\end{array}$ \\
\hline A.L.C. & $\begin{array}{c}1.01 \pm 0.09 \\
(1.00 \div 0.89)\end{array}$ & $\begin{array}{r}0.87 \\
10.78\end{array}$ & $\begin{array}{l}+0.06 \\
-0.93)\end{array}$ \\
\hline A. 0 . & $\begin{array}{l}0.56 \pm 0.07 \\
(0.48 \div 0.63)\end{array}$ & $\begin{array}{r}0.56 \\
(0.52\end{array}$ & $\begin{array}{l}+0.05 \\
\pm 0.63)\end{array}$ \\
\hline A.L.O. & $\begin{array}{c}0.81 \pm 0.03 \\
(0.78 \div 0.85)\end{array}$ & $\begin{array}{r}0.71 \\
10.67\end{array}$ & $\begin{array}{l} \pm .04 \\
\pm 0.74)\end{array}$ \\
\hline A.F. & $\begin{array}{c}0.61 \pm 0.08 \\
(0.52-0.70)\end{array}$ & $\begin{array}{r}0.59 \\
(0.56\end{array}$ & $\begin{array}{l}+0.03 \\
\pm 0.03)\end{array}$ \\
\hline L.A. & $\begin{array}{c}0.40 \pm 0.05 \\
(0.33 \div 0.44)\end{array}$ & $\begin{array}{r}0.32 \\
10.30\end{array}$ & $\begin{array}{l}+0.03 \\
\pm 0.37)\end{array}$ \\
\hline L.C. & $\begin{array}{c}0.34 \pm 0.03 \\
(0.30 \div 0.37)\end{array}$ & $\begin{array}{r}0.36 \\
10.30\end{array}$ & $\begin{array}{l}+0.03 \\
\pm 0.37)\end{array}$ \\
\hline L.T. & $\begin{array}{l}1.67 \pm 0.08 \\
(1.59 \div 1.78)\end{array}$ & $\begin{array}{r}1.47 \\
(1.33\end{array}$ & $\begin{array}{l}+0.10 \\
\pm=1.55)\end{array}$ \\
\hline L.A.L. & $\begin{array}{c}3.52 \pm 0.08 \\
(3.40 \div 3.59)\end{array}$ & $\begin{array}{r}3.25 \\
(2.70\end{array}$ & $\begin{array}{l} \pm 0.36 \\
-3.70)\end{array}$ \\
\hline A.A. & $\begin{array}{l}1.64 \pm 0.07 \\
(1.55 \div 1.70)\end{array}$ & $\begin{array}{r}1.19 \\
(1.07\end{array}$ & $\begin{array}{l}+0.13 \\
-1.37)\end{array}$ \\
\hline L.A.B. & $\begin{array}{l}2.28 \pm 0.70 \\
(1.30 \div 2.78)\end{array}$ & $\begin{array}{r}1.24 \\
(1.11\end{array}$ & $\begin{array}{l}+0.11 \\
-1.41)\end{array}$ \\
\hline L.F.O. & $\begin{array}{l}1.21 \pm 0.14 \\
(1.01 \div 1.24)\end{array}$ & $\begin{array}{r}0.71 \\
(0.63\end{array}$ & $\begin{array}{l}+0.08 \\
-0.81)\end{array}$ \\
\hline L. 0 . & $\begin{array}{l}1.63 \pm 0.07 \\
(1.53=1.76)\end{array}$ & $\begin{array}{r}0.98 \\
(0.94\end{array}$ & $\begin{array}{l}+0.05 \\
-1.06)\end{array}$ \\
\hline
\end{tabular}

$\mathrm{N}=$ Número de Individuos analizados

Tabla 1 - Mediciones corporales de hembras de $T$. foliosi y $T$. footei. En cada medición se incica el promedio, desviación estandar y rango.

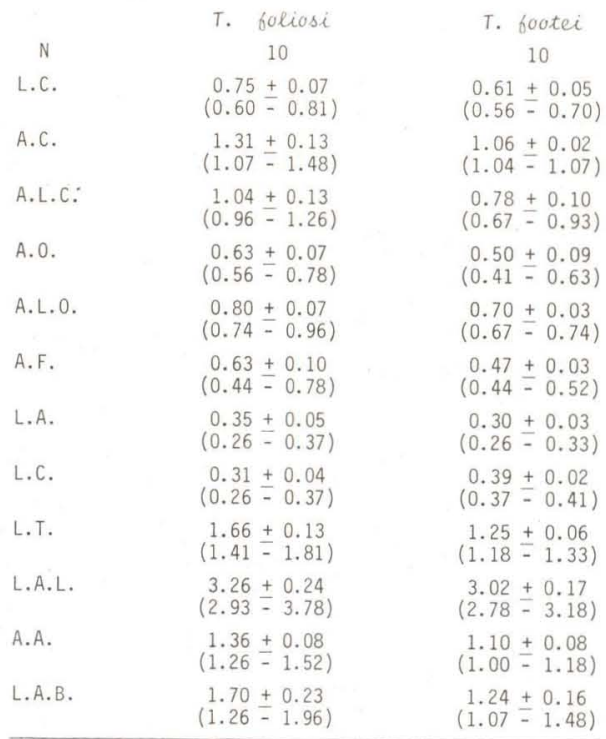

$N=$ Número de Individuos Analizados

Tabla 2 - Mediciones corporąles de machos de $T$. foliosi y $T$. footei. En cada medición se indica el promedio, desviación estandar y rango. 


\section{Genitalia}

Machos: epandrio negro (Fig. 21). Aedeagus y apodema eyaculador como en las Figs. 25 y 29. Hembras: un par de espermatecas café (Fig. 33). Ovipositor café, aguzado en al ápice (Fig. 37).

Huevos

Blancos (Fig. 41).

Larvas

Blanquecinas (Fig. 43). Ganchos mandibulares como en la Fig. 46. Espiráculos posteriores como en la Fig. 49.

Pupas

Ovoides, negras (Fig. 52).

Alas

Modelo alar como en la Fig. 55. Segunda célula costal gris, vena subcostal, vena humeral y vena $r_{1}$ café oscura.

\section{Cromosomas}

6 pares. Ausencia de un par sexual heteromórfico (Figs. 59, 60, 64 y Tabla 5).

Hospedero

Haplopappus foliosus D. C. (Compositae). Las hembras oviponen en el interior de las flores y las larvas se desarrollan en los ovarios.

Esta especie se nominó en relacion a su hospedero $H$. foliosus en el cual desarrolla su ciclo vital.

Trupanea chrysanthemifolii, sp. n.

(Figs. 2, 6, 10, 15, 16, 22, 26, 30, 34, 38, 44, 47, 50, 53, 56, 61, 62, 65)

Tipos: holotipo hembra, Valparaíso (Mirasol) 3 de Febrero de 1981. Paratipos 5 hembras, 5 machos, Valparaíso (Mirasol, Algarrobo) 15 de febrero de 1979.

Descripción

Las mediciones corporales de los adultos se indican en las Tablas 3 y 4. Los datos cromosómicos se muestran en la Tabla 5.

\section{Cabeza}

Cara amarilla; frente café; triángulo ocelar gris. Cuatro pares de cerdas frontoorbitales inferiores. Dos pares de cerdas fronto-orbitales superiores siendo el par posterior blanco y mas corto que el par anterior. Cerdas verticales internas de color negro. Cerdas verticales externas, post-ocelares, post-verticales y postoculares de color blanco. Márgenes de la región post-ocular amarillos con la región central negra (Fig. 2). Antenas como en la Fig. 6.

Tórax

Gris, cubierto de pequeñas cerdas blancas, cerdas escapulares y propleurales blancas. Un par de cerdas acrosticales. Un par de cerdas dorsocentrales. Dos cerdas escutelares. Dos cerdas notopleurales siendo la posterior blanquecina y mas corta que la anterior. Mesopleuron cubierto de cerdas pequeñas blancas, una cerda mesopleural negra. Cerdas pteropleurales blancas. Esternopleuron cubierto de pequeñas cerdas blancas; una cerda esternopleural (Fig. 10).

\section{Abdómen}

Gris, cubierto de pequeñas cerdas blancas. Hembras: en el último tergito abdominal con tres pares de cerdas negras. Machos: en el último tergito abdominal con 3 a 4 pares de cerdas negras. En las hembras, funda del ovipositor negro cubierto de pequeñas cerdas blancas, excepto en la parte apical en donde está cubierto de pequeñas cerdas negras (Fig. 15 y 16). 


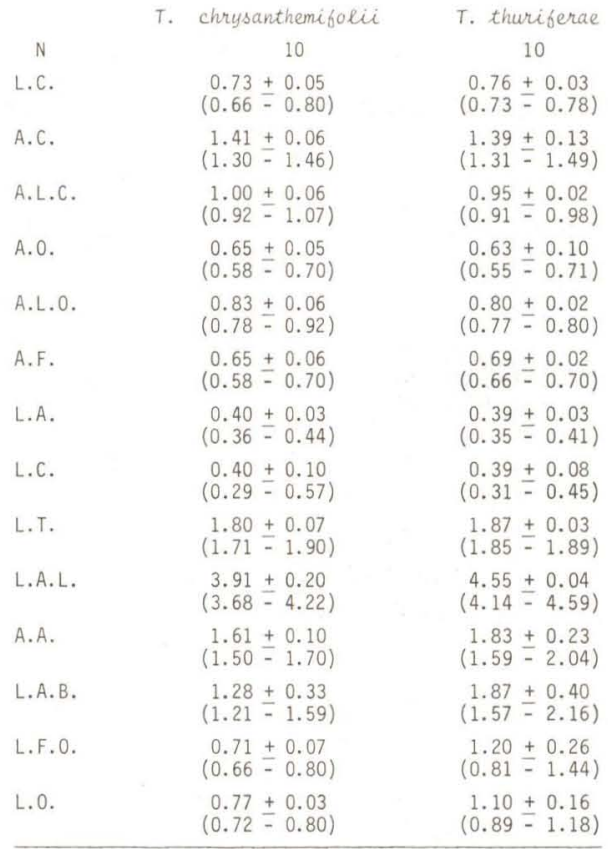

$\mathrm{N}=$ Número de Individuos analizados

Tabla 3 - Mediciones corporales de hembras de $T$. chrysanthemifolii y $T$. thuriferae. En cada medición se indica el promedio, desviación estandar y rango.

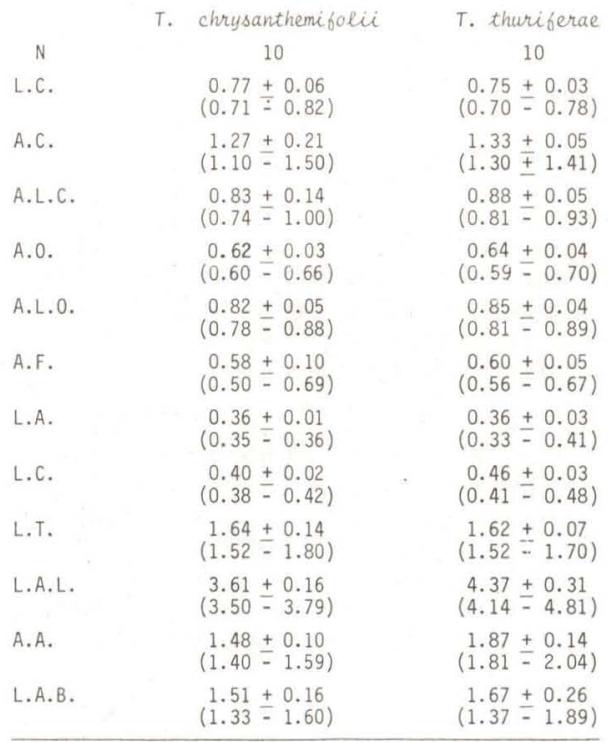

$\mathrm{N}=$ número de Individuos analizados.

Tabla 4 - Mediciones corporales de machos de $T$. chrysanthemifolii y $T$. thuriferae. En cada medición se indica el promedio, desviación estandar y rango. 


\section{Patas}

Amarillas. Coxas cubiertas de pequeñas cerdas blancas, femur, tibia y tarsos provistos de cerdas negras.

\section{Genitalia}

Machos: epandrio negro (Fig. 22). Aedeagus y apodema eyaculador como en las Figs. 26 y 30 . Hembras: un par de espermatecas café (Fig. 34). Ovipositor café, aguzado en el ápice (Fig. 38).

Larvas

Blancas (Fig. 44). Ganchos mandibulares como en la Fig. 47. Espiráculos posteriores como en la Fig. 50.

\section{Pupas}

Ovoides, negras (Fig. 53).

Alas

Diseño alar como en la Fig. 56. Segunda célula costal hialina, vena subcostal, vena humeral y vena $r_{1}$ amarillas (Fig. 56).

Cromosomas

6 pares. Ausencia de un par de heteromórficos (Figs. 61, 62, 65 y Tabla 5).

Hospedero

Las hembras oviponem en las flores de Haplopappus chrysanthemifolius (Less.) (Compositae) y las larvas se alimentan de los ovarios.

Esta espécie se nominó en relación a su hospedero $H$. chrysanthemifolius en el cual desarrolla su ciclo vital.

\section{Trupanea footei, sp. n.}

(Figs. 3, 7, 11, 17, 18, 23, 27, 31, 35, 39, 42, 45, 48, 51, 54, 57)

Tipos: holotipo hembra. Rapel, 15 de Marzo de 1981. Paratipos, 8 hembras y 7 machos, Rapel, 15 de Marzo de 1981.

Descripción

Mediciones corporales de los adultos se indican en las Tablas 1 y 2 .

\section{Cabeza}

Cara amarilla, frente café. Triángulo ocelar gris. Lámina para-frontal gris con algunos pequeñas cerdas blancas. Región post-orbital gris en el centro con las regiones laterales amarillas. Cerdas verticales internas de color negro. Cerdas post-ocelares, verticales externas, post-verticales y post-oculares blancas (Fig. 3). Antenas como en la Fig. 7.

\section{Tórax}

Gris, cubierto en la parte dorsal de pequeñas cerdas blancas, cerdas escapulares y propleurales blancas. Un par de cerdas dorsocentrales. Escutelo gris con algunas cerdas pequeñas blancas en los márgenes. Un par de cerdas escutelares. Dos cerdas notopleurales, la posterior blanca y más pequeñas que el anterior. Mesopleuron cubierto de cerdas blancas y una cerda negra mesopleural. Pteropleuron tapizado de cerdas blancas. Esternopleuron cubierto de cerdas blancas y una cerda negra esternopleural (Fig. 11).

\section{Abdómen}

Gris, cubierto en la parte dorsal de pelos blancos. En las hembras, la funda del ovipositor negra con pelos blancos, excepto en la parte posterior. Tanto en hembras como en machos, último tergito abdominal provisto de tres pares de cerdas negras (Figs. 17 y 18).

\section{Patas}

En las hembras y machos las patas son totalmente amarillas. 
Genitalia

Machos: protandrio gris, epandrio negro, proctiger amarillo (Fig. 23). Aedeagus y apodema eyaculador como en las Figs. 27 y 31. Hembras: un par de espermatecas levemente pigmentadas de color café (Fig. 35), ovipositor como en la Fig. 39.

\section{Huevos}

Blanquecinos (Fig. 42).

\section{Larvas}

Blanco amarillentas (Fig. 45). Ganchos mandibulares como en la Fig. 48. Espiráculos posteriores como en la Fig. 51.

Pupas

Negras (Fig. 54).

Alas

Diseño alar como en la Fig. 57.

\section{Hospedero}

Las hembras ponen sus huevos en las flores de Haplopappus sp. (Compositae) y las larvas se alimentan de los ovarios.

Esta especie es dedicada al Dr. Richard Foote, especialista mundial en Tephritidae.

\section{Trupanea thuriferae, sp. n.}

(Figs. 4, 8, 12, 19, 20, 24, 28, 32, 36, 40, 58, 63, 66)

Tipos: holotipo hembra, Santiago (Cerro San Chistóbal), 10 de Noviembre de 1978. Paratipos, 2 machos, 1 hembra, 10 de Noviembre de 1978, Santiago (Cerro San Cristóbal).

\section{Descripción}

Las medidas corporales de los adultos se indican en las Tablas 3 y 4 . Los datos cromosómicos se indican en la Tabla 5 .

\section{Cabeza}

Café, frente café oscura. Triángulo ocelar gris oscuro. Región post-ocular gris oscura. Láminas para frontales grises provistas de una corrida de pequeñas cerdas blancas. Tres pares de cerdas fronto-orbitales inferiores. Dos pares de cerdas fronto-orbitales superiores, el posterior más corto y de color blanco. Cerdas verticales internas negras. Cerdas post-ocelares, cerdas verticales internas y cerdas post-oculares blancas (Fig. 4). Antenas como en la Fig. 8.

\begin{tabular}{|c|c|c|c|c|c|c|c|c|c|c|}
\hline & \multirow[b]{2}{*}{ N } & & \multicolumn{3}{|c|}{ PARES DE } & \multicolumn{2}{|c|}{ CROMOSOMAS } & \multirow[b]{2}{*}{ VI } & \multirow[b]{2}{*}{ LTC } & \multirow[b]{2}{*}{ s } \\
\hline & & & $I^{*}$ & 11 & 111 & IV & $v$ & & & \\
\hline \multirow[b]{2}{*}{ T. foliose } & \multirow[t]{2}{*}{15} & \multirow{2}{*}{\begin{tabular}{l}
\multicolumn{1}{c}{${ }^{r}$} \\
LONGITUD (इ) \\
MORFOLOGIA \\
CROMOSOMICA
\end{tabular}} & $\begin{array}{r}2.3 \\
11.3\end{array}$ & $\begin{array}{r}1.3 \\
16.5\end{array}$ & $\begin{array}{r}1.2 \\
18.0\end{array}$ & $\begin{array}{r}1.7 \\
19.8\end{array}$ & $\begin{array}{r}1.3 \\
21.5\end{array}$ & $\begin{array}{r}1.1 \\
24.1\end{array}$ & \multirow{2}{*}{\multicolumn{2}{|c|}{$36.0 \pm 7.4$}} \\
\hline & & & $S M$ & M & M & M & M & M & & \\
\hline \multirow{3}{*}{$\begin{array}{l}\text { T. chrysanthe } \\
\text { mifole }\end{array}$} & \multirow[t]{3}{*}{10} & $r$ & 1.4 & 1.3 & 1.6 & 1.4 & 1.5 & 1.4 & \multirow{3}{*}{\multicolumn{2}{|c|}{$30.5 \pm 3.1$}} \\
\hline & & LONGITUD ( $E$ ) & 10.7 & 15.8 & 17.0 & 17.8 & 19.0 & 19.7 & & \\
\hline & & $\begin{array}{l}\text { MORFOLOGIA } \\
\text { CROMOSOMICA }\end{array}$ & M & $M$ & M & M & M & M & & \\
\hline \multirow{3}{*}{ T. thuriferae } & \multirow[t]{3}{*}{10} & $r$ & 3.3 & 1.5 & 2.0 & 2.0 & 1.8 & 1.9 & \multirow{3}{*}{34.3} & \\
\hline & & LONGITUD ( $\%$ ) & 12.2 & 14.4 & 15.1 & 16.1 & 20.6 & 21.7 & & \pm 4.8 \\
\hline & & CROMOSOMICA & SM & M & SM & $S M$ & SM & SM & & \\
\hline
\end{tabular}

Tabla 5 - Número y morfología cromosómica en $T$. foliosi, $T$. chrysanthemifolii y $T$. thuriferae. 
Tórax

Gris, cubierto con pequeñas cerdas blancas. Un par de cerdas dorsocentrales. Un par de cerdas acrosticales. Escutelo con algunas cerdas blancas en la parte apical y provisto de un par de cerdas escutelares. Cerditas propleurales blancas. Dos cerdas notopleurales, siendo la posterior más corta y blanca. Una cerda mesopleural. Una cerda pteropleural. Una cerda esternopleural (Fig. 12).

\section{Abdómen}

Gris, cubierto de cerdas blancas. En las hembras funda del ovipositor negro, en la parte apical negro billante y desprovido de cerdas (Fig. 19 y 20).

Patas

Tanto en machos como en hembras totalmente amarillas, cubiertas con cerdas amarillas.

\section{Genitalia}

Machos: protandrio gris (Fig. 24). Aedeagus y apodema eyaculador como en las Figs. 28 y 32 respectivamente. Hembras: un par de espermatecas sin pigmentación (Fig. 35). Ovipositor como en la Fig. 40.

Alas

Hialinas basalmente, con manchas y bandas oscuras que comienzan a nivel de la vena radio medial cruzada. Célula cubital anterior totalmente hialina.

\section{Cromosomas}

Seis pares de cromosomas. Par sexual isomórfico (Figs. 63, 66 y Tabla 5). Hospedero

Las hembras oviponen en las flores de Flourensia thurifera (Mol.) D. C. (Compositae) y las larvas se desarrolan en los ovarios.

Esta especie se nominó en relación a su hospedero, $F$. thurifera.

\section{CLAVE PARA DISTINGUIR LAS ESPECIES DE TRUPANEA DESCRITAS EN ESTE TRABAJO}

1. Alas hialinas basalmente. Con manchas y bandas oscuras que comienzan a nivel de la vena radio medial cruzada. Células cubital anterior totalmente hialina ...................... Trupanea thuriferae Alas hialinas basalmente con manchas y bandas oscuras que comienzan

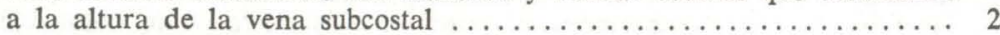

2. 2a. célula costal gris, vena sub-costal, vena humeral y vena $r_{2}$ café

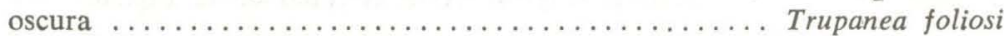
2.a célula costal hialina. Vena sub-costal, vena humeral y vena $r_{2}$

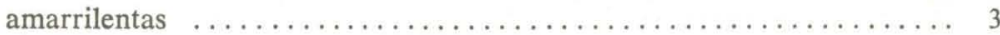

3. Longitud del ovipositor sobrepasa la mitad de la longitud del abdó-

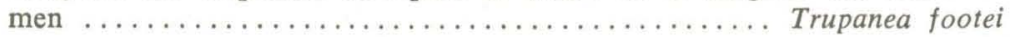

4. Longitud del ovipositor no sobrepasa la mitad de la longitud del abdómen ..................... Trupanea chrysanthemifolii

\section{DISCUSIÓN}

Trupanea foliosi es muy similar morfológicamente con respecto a Trupanea chrysanthemifolii. Sin embargo estas especies se diferencian entre sí por los siguientes caracteres: la funda del ovipositor y ovipositor en las hembras de $T$. foliosi son de una longitud mayor que en las hembras de $T$. chrysanthemifolii. La genitalia en los machos de ambas especies también es diferente. En relación al alla, en $T$. foliosi la segunda célula costal es gris, en cambio en $T$. chrysanthemifolii es hialina. La vena sub-costal, vena humeral y vena $r_{2}$ en $T$. foliosi son café oscuras, en cambio en $T$. chrysanthemifolii son amarillas. 

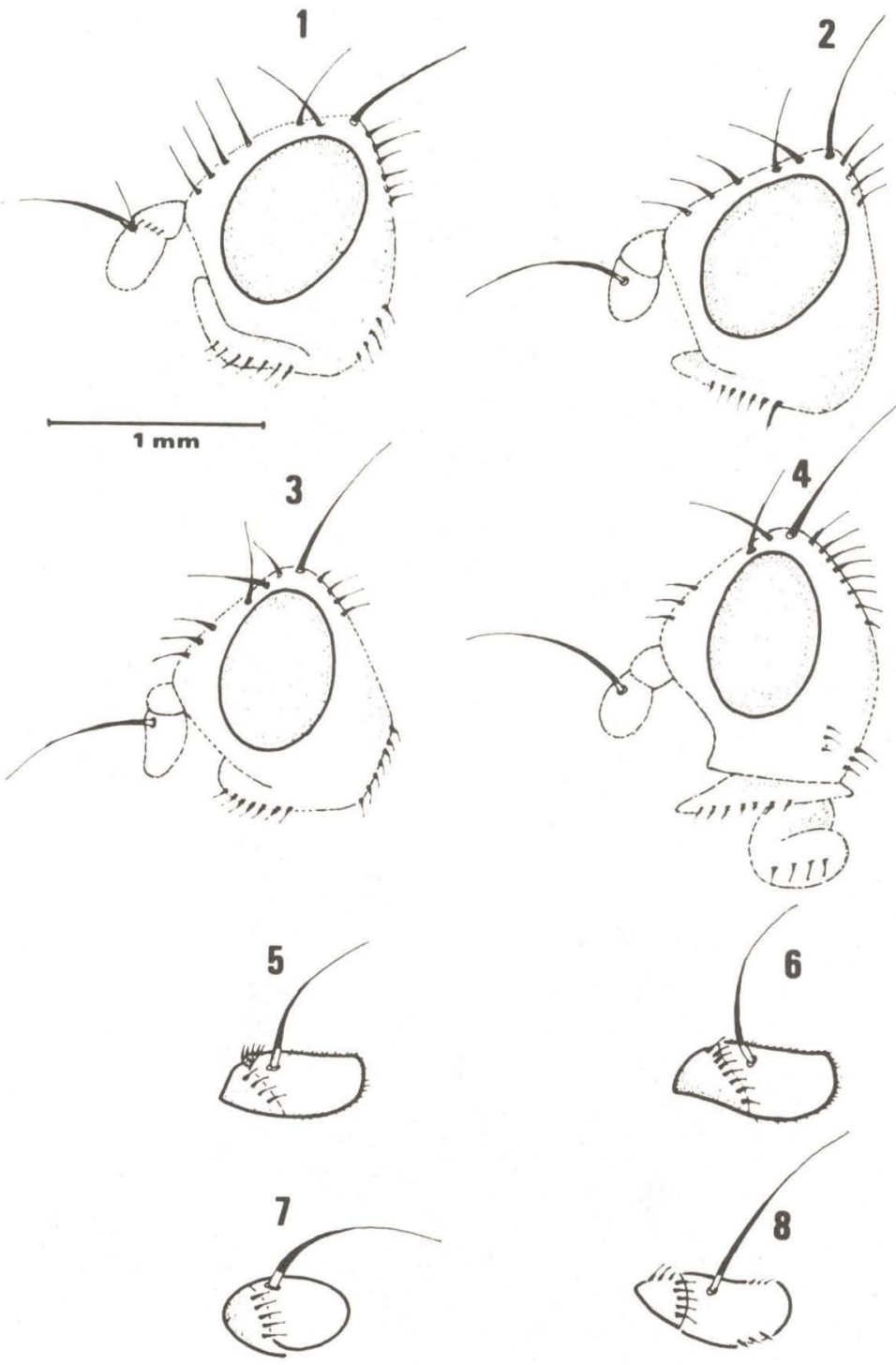

$1 \mathrm{~mm}$

Figuras 1-4 - Vista lateral de cabezas: 1. T. foliosi; 2. T. chrysanthemifolii; 3. T. footei; 4. T. thuriferae.

Figuras 5-8 - Vista lateral de antenas: 5. T. foliosi; 6. T. chrysanthemifolii; 7. T. footei; 8. T. thuriferae. 

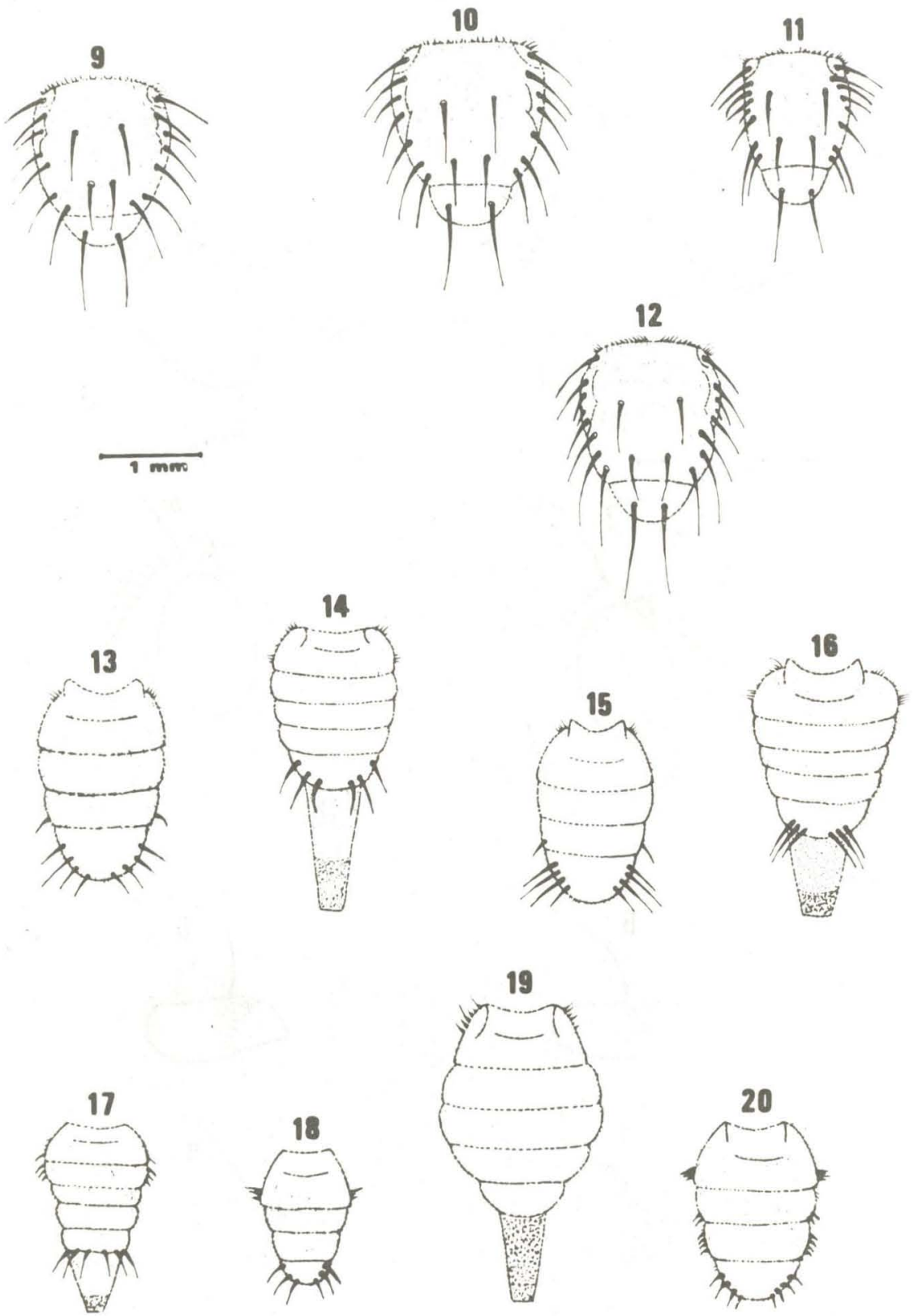

Figuras 9-12 - Vista dorsal de tórax: 9. T. foliosi; 10. T. chrysanthemifolii; 11. T. footei; 12. T. thuriferae.

Figuras 13-20 - Vista dorsal de abdómenes: 13. macho T. foliosi; 14. hembra $T$. foliosi; 15 . macho $T$. chrysanthemifolii; 16 . hembra $T$. chrysanthemifolii; 17. hembra $T$. footei; 18 . macho $T$. footei; 19 . hembra $T$. thuriferae; 20. macho $T$. thuriferae. 

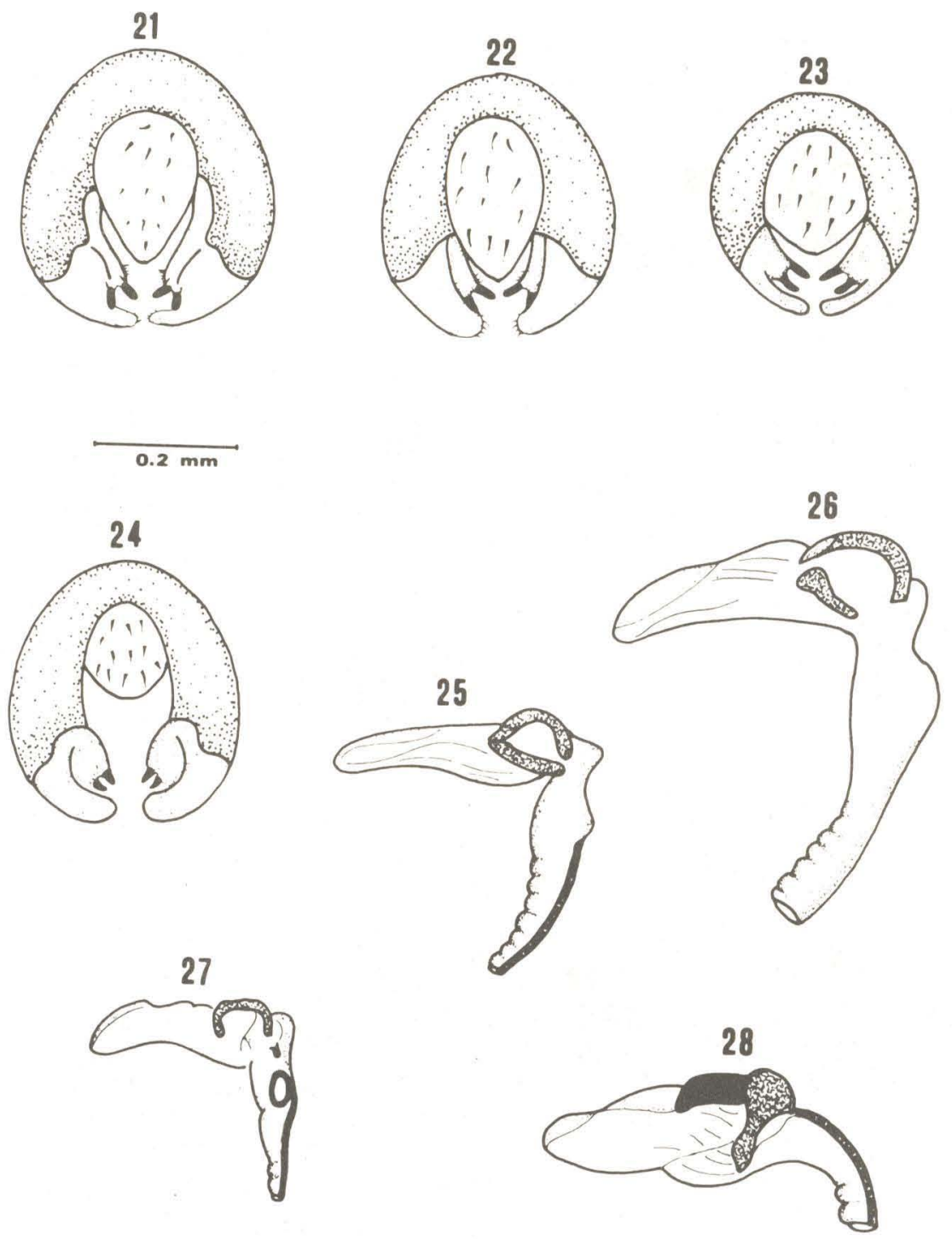

Figuras 21-24 - Vista posterior de Genitalia de macho:

21. T. foliosi; 22. T. chrysanthemifolii; 23. T. footei; 24. T. thuriferae.

Figuras 25-28 - Aedeagus:

25. T. foliosi; 26. T. chrysanthemifolii; 27. T. footei; 28. T. thuriferae. 

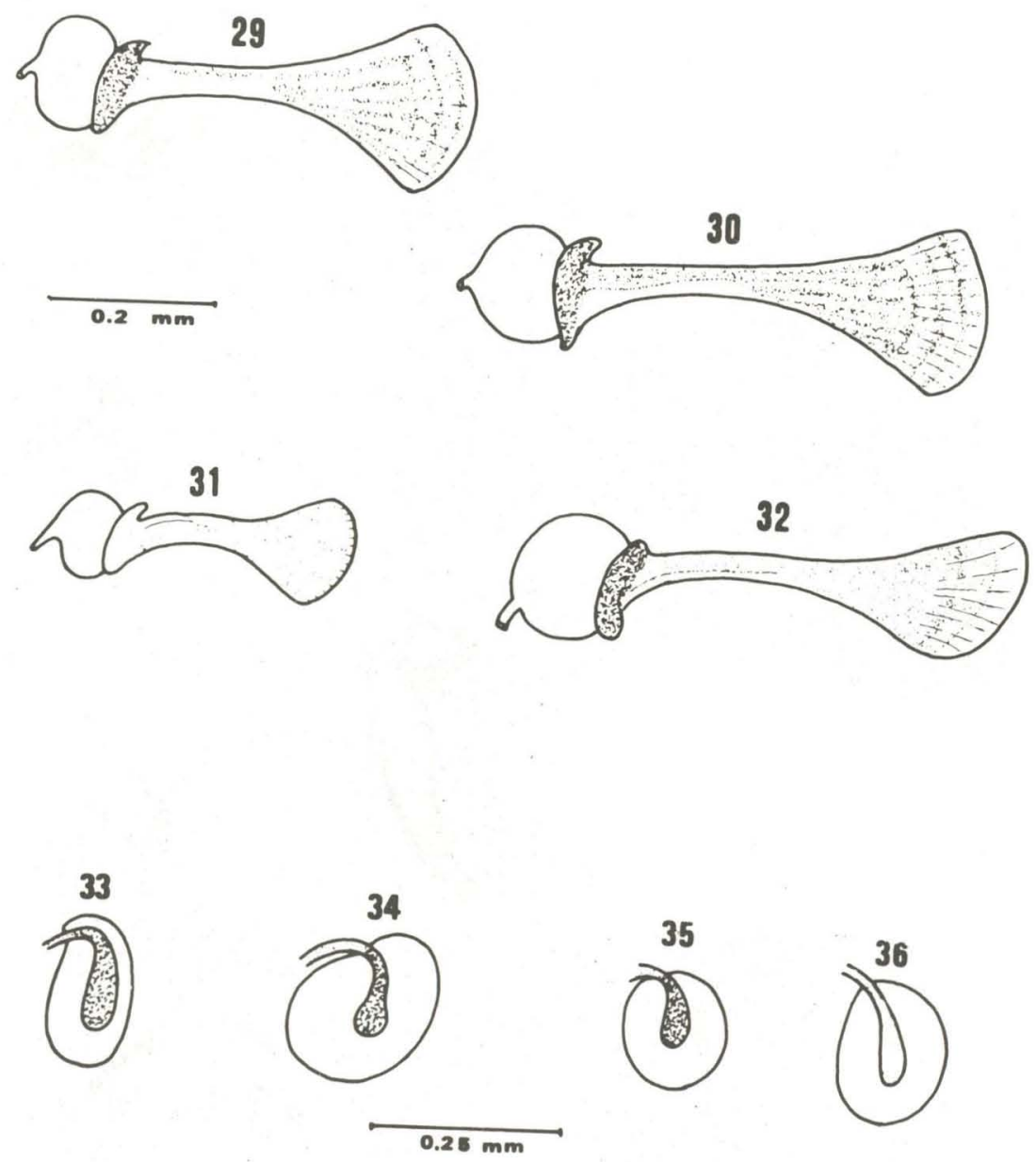

Figuras 29-32 - Apodemas eyaculadores:

29. T. foliosi; 30. T. chrysanthemifolii; 31. T. footei; 32. T. thuriferae.

Figuras 33-36 - Espermatecas:

33. T. foliosi; 34. T. chrysanthemifolii; 35. T. footei; 36. T. thuriferae. 

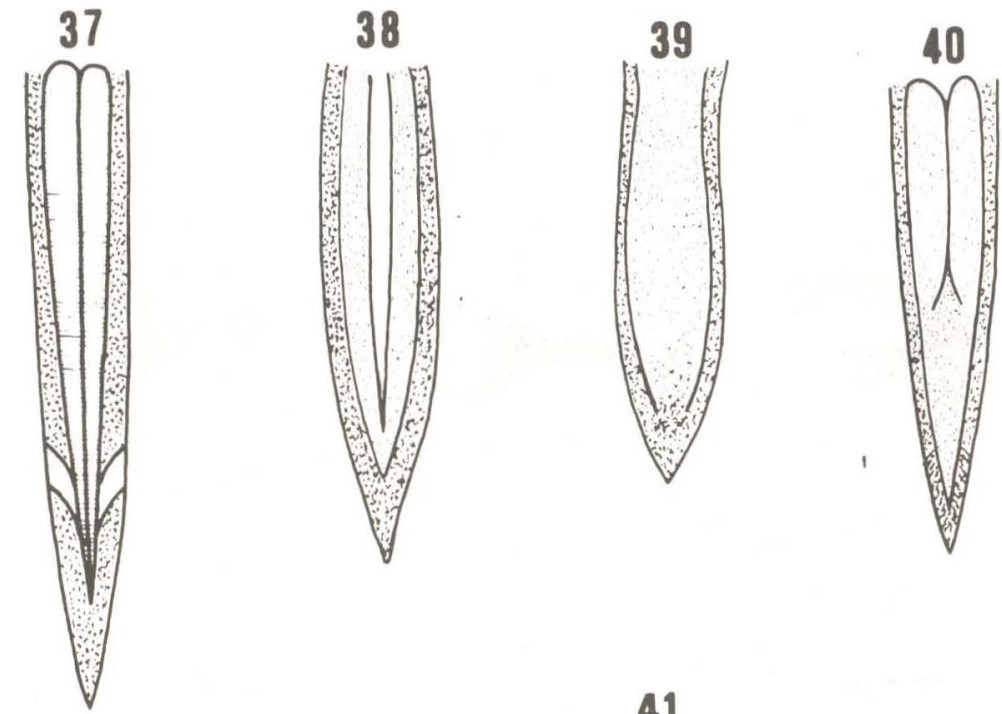

$1 \mathrm{~mm}$
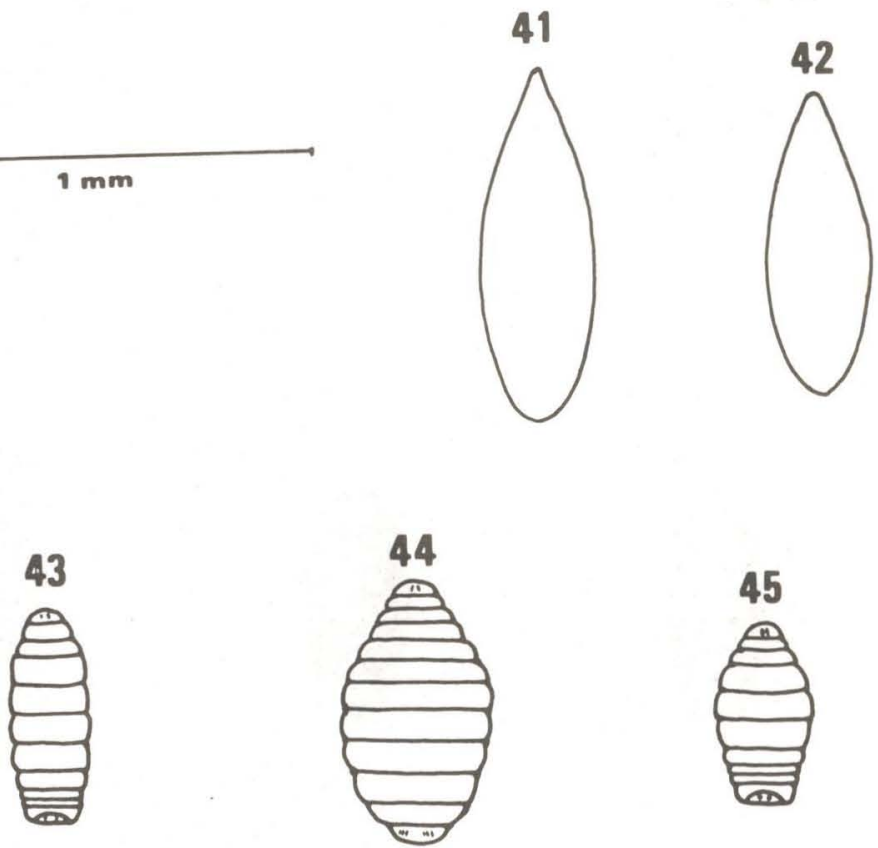

Figuras $37-40$ - Ovipositores:

37. T. foliosi; 38. T. chrysanthemifolii; 39. T. footei; 40. T. thuriferae.

Figuras 41-42 - Huevos:

41. T. foliosi; 42. T. footei.

Figuras 43-45 - Larvas:

43. T. foliosi; 44. T. chrysanthemifolii; 45. T. footei. 

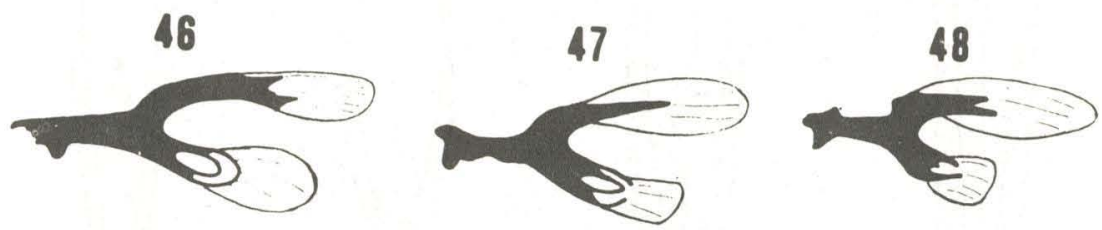

$0.2 \mathrm{~mm}$
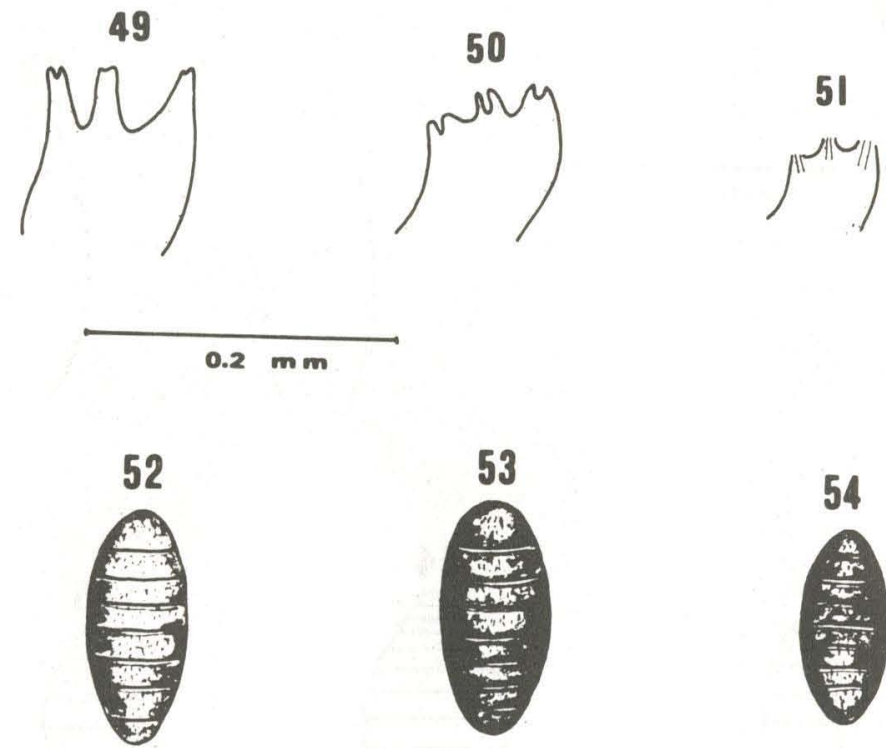

$1 \mathrm{~mm}$

Figuras 46-48 - Ganchos mandibulares de larvas:

46. T. foliosi; 47. T. chrysanthemifolii; 48. T. footei.

Figuras 49-51: Espiráculos posteriores de larvas:

49. T. foliosi; 50. T. chrysanthemifolii; 51. T. footei.

Figuras 52-54 - Pupas:

52. T. foliosi; 53. T. chrysanthemofilii; 54. T. footei. 

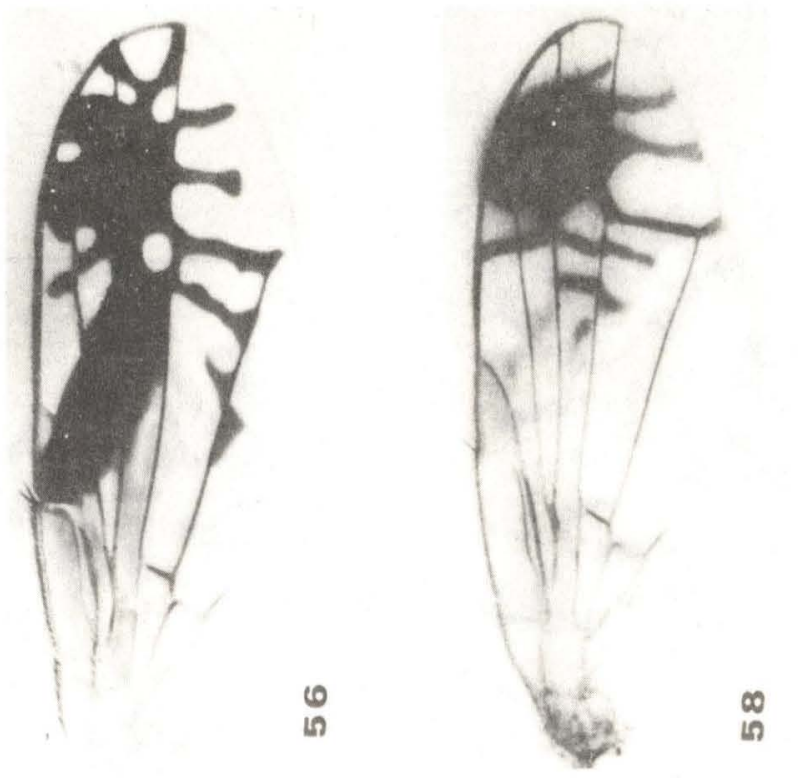

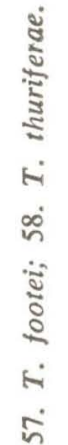
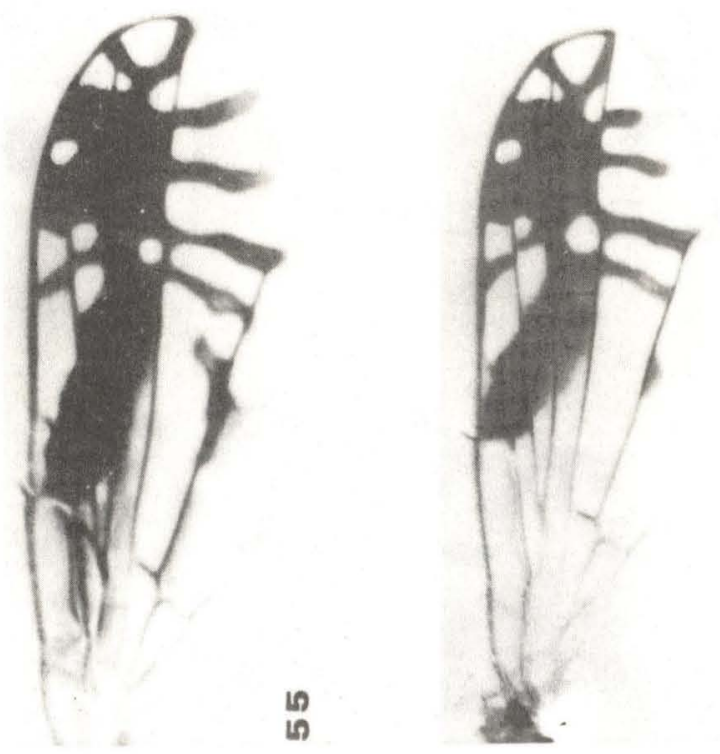

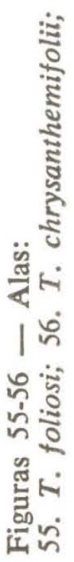

เก

is 


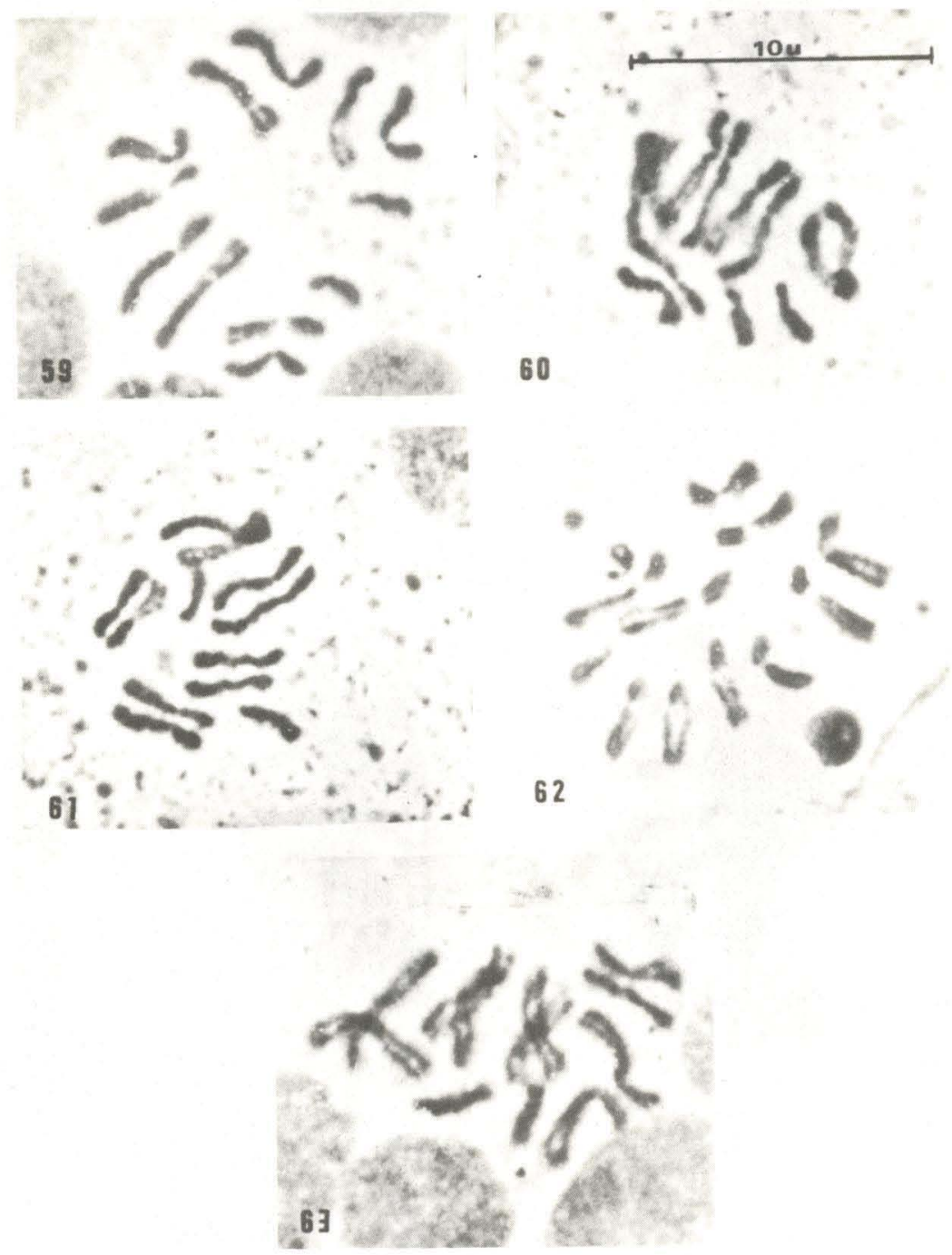

Figuras 59-63 - Cromosomas metafásicos:

59. hembra $T$. foliosi; 60 . macho $T$. foliosi; 61 . hembra $T$. chrysanthemofilii; 62. macho $T$. chrysanthemofilii; 63. macho $T$. thuriferae. 

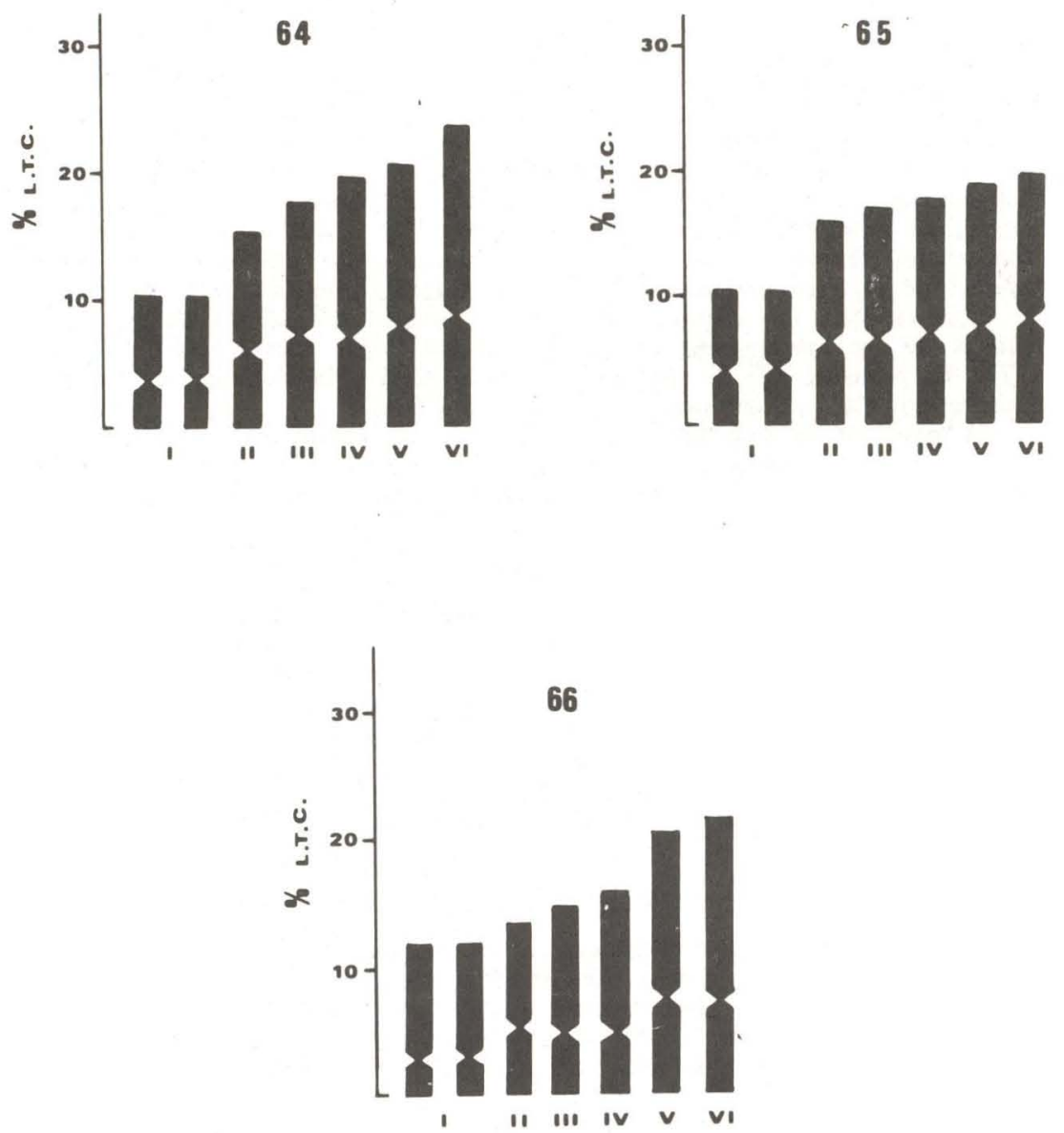

Figuras 64-66 - Idiogramas:

64. T. foliosi; 65. T. chrysanthemofilii; 66. T. thuriferae.

La morfología de las larvas y los espiráculos posteriores de las mismas son bastantes diferentes entre estas dos especies. Respecto a los cromosomas, el par I en $T$. foliosi es sub-metacentrico, en cambio en $T$. chrysanthemifolii es metacentrico.

$T$. footei se diferencia de $T$. foliosi y $T$. chrysanthemifolii en que es mas pequeña corporalmente. Además existen notables diferencias en la genitalia.

Con respecto a $T$. thuriferae, esta especie se diferencia morfológicamente de $T$. foliosi, $T$. chrysanthemifolii y $T$. footei en los siguientes caracteres: en $T$. thuriferae las alas son hialinas basalmente,. con manchas y bandas oscuras que comienzan a nivel de la vena radio medial cruzada. La célula cubital anterior es totalmente hialina. En $T$. foliosi, $T$. chrysanthemifolii y T. footei las alas son hialinas basalmente con manchas y bandas oscuras que comienzan a la altura de la vena sub-costal. Además, la genitalia de esta especie, tanto en machos como en hembras es bastante diferente de T. foliosi, T. chrysanthemifolii y $T$. footei. 
En relación a los cromosomas se observa que, excepto en $T$. footei, especie en la cual no se describe el cariotipo, $T$. foliosi, $T$. chrysanthemifolii y $T$. thuriferae presentan el mismo número cromosómico $(2 \mathrm{n}=12)$. En $T$. foliosi y $T$. chrysanthemifolii la morfología cromosómica es bastante similar, presentando una tendencia a la metacentría exceptuando el par I de $T$. foliosi que es sub-metacéntrico. En $T$. thuriferae todos sus cromosomas son submetacéntricos, con excepción del par II que es metacéntrico. Además, es importante resaltar que en todas estas especies los cromosomas sexuales son isomórficos. Situaciones de esta naturaleza han sido descritas para otras especies de dípteros pertenencientes a otras familias. Sin embargo, en algunas de estas especies los cromosomas sexuales pueden ser identificados por el análisis de los cromosomas politénicos (Acton 1957, Martin 1962, Amabis 1977). Es probable que al analizar los cromosnmas politénicos de las especies descritas en este trabajo ocurra una situación similar.

A pesar de la semejanza morfológica de los cromuzomas sexuales con los autosomas, en cada especie el par sexual fué distinguido pur presentar una marcada heteropicnósis positiva y además por su falta de apareamiento en las placas metafásicas.

Con respecto a la biología, todas las especies descritas se asocian a plantas de la familia Compositae, de manera similar a todas las especies de Trupanea en las cuales se han descrito sus plantas huéspedes (Wasbauer 1972, Foote 1967). Además con excepción de $T$. thuriferae que se asocia a Flourensia thurifera (Mol.) D. C., todas las especies descritas en este trabajo viven asociadas a plantas del género Haplopappus. Las hembras de estas especies, incluyendo $T$. thuriferae ponen sus huevos en las inflorecencias de sus plantas huéspedes y las larvas se alimentan de los ovarios. Una vez que las larvas completan su desarrollo, pupan en el interior de las flores, de donde nacen posteriormente los adultos, completándose de esta manera su ciclo vital.

\section{AGRADECIMIENTOS}

Deseo expresar mi agradecimiento al Dr. Richard Foote por sus estímulos para que publicara este trabajo. Agradezco además a los Drs. Aldo Malavasi, J. S. Morgante y Sergio Matioli por la invitación que me hicieron para visitar el Depto. de Biologia de la USP en donde gracias a su colaboración finalicé este estudio. Mis agradecimientos también al Dr. Raúl Cortés por la lectura del manuscrito y sus valiosas sugerencias.

Este trabajo fue realizado a través del proyecto N 1195-8283, Universidad de Chile y convenio CONICYT/CNPq, processo n. ${ }^{\circ} 2.31 .20 .018 / 82$.

\section{REFERENCIAS}

Acton, A. B., 1957. Sex chromosome inversion in Chironomus. Amer. Natur. 91: $57-59$.

Aczél, M., 1949. Catálogo de la familia Trypetidae de la región Neotropical. Acta zool. Lilloana 7: 177-328.

Aczél, M., 1953. The genus Trupanea in the Neotropical region 2. The argentina group. Dusenia 4: 365-387.

Amabis, J. M., 1977. Cytological evidence of male heterogamety in sex determination of Telmatoscopus albipunctatus (Diptera, Psychodidae). Chromosoma 62: 133-138.

Boyes, J. W., J. M. van Brink \& C. Boyes, 1971. Chromosomes of Syrphinae (Diptera, Syrphidae). Misc. Publ. genet. Soc. Canada: 158 p.

Brèthes, J., 1908. El gnero Urellia (Diptera) en el Plata. Ann. Mus. nac. Buenos Aires 16: 367-374.

Bush, G. L., 1966. The taxonomy, cytology and evolution of genus Rhagoletis in North America (Diptera, Tephritidae). Bull. Mus. comp. Zool. 134(11): 431-562.

Foote, R. H., 1967. A catalogue of the Diptera of the Americas South of the United States. Depto. de Zoologia, Secretaria da Agricultura, S. Paulo 57: 1-91. 
Foote, R. H., 1980. Fruit fly genera south of the United States. U. S. Department of Agriculture, Tech. Bulletin 1600, 79 p.

Hendel, F., 1914. Die Bohrfliegen Südamerikas. K. zool. anthrop. ethnogr. Mus. Abhandl. Ber. 14(3): 1-84.

Hering, E. M., 1936. Neue südamerikanische Trypanea-arten (13. Beitrag zur Kenntniss der Trypetidae). Revta Ent., Rio de Janeiro 6: 527-332.

Hering, E. M., 1941. Trypetidae, Beiträge zur Fauna Perus 1: 121-176.

Hering, E. M., 1942. Neue Gattungen und Arten palaearktischer und exotischer Fruchtfliegen. Siruna Seva 4: 1-31.

Levan, A., K. Fredga \& A. Sandberg, 1964. Nomenclature of centromeric position on chromosome. Hereditas 52: 201-220.

Macquart, J., 1843. Diptères exotiques nouveaux ou peu connus. Mém. Soc. Roy. Sci., Agric., Arts Lille (1842): $162-460$ (published separately as vol. 2 pt. 3 , pp. 4-304).

Martin, J., 1962. The interrelation of inversion systems in the midge Chironomus intestinctus (Diptera, Nematocera). Aust. J. biol. Sci. 15: 166-173.

Martin, J., 1966. Female heterogamety in Polypedilum nubifer (Diptera Nematocera). Am. Nat. 100: 157-159.

Malloch, J. R., 1933. Acalyptrata, pp. 177-391 (fac. 4), in British Museum (Natural History): Diptera of Patagonia and South Chile 6: 499.

Munro, H. K., 1964. The genus Trupanea in Africa: an analytical study in biotaxonomy. Memoir So. Afr. Dept. Agric., Tech. Serv. 8: 101 p.

Schiner, I. R., 1868. Diptera (art. 1), 338 pp., in Reise der österreichische Fregatte Novara um die Erde, Zool., 2 (Abt. 1, sect. B) Wien.

Stuardo, O. C., 1946. Catalogo de los Dipteros de Chile, 250 p. Ministerio de Agricultura, Santiago.

Wasbauer, M. S., 1972. An annotated host catalog of the fruit flies of America North of Mexico (Diptera, Tephritidae). Laboratory Services Entomology. Bureau of Entomology, Dep. Agric. Sacramento. Occas. Papers 19: 1-171. 